\title{
Correction to: PTEN Expression in Human Granulosa Cells Is Associated with Ovarian Responses and Clinical Outcomes in IVF
}

\author{
Jianfeng Yao ${ }^{1,2} \cdot$ Rongfu Huang ${ }^{3} \cdot$ Ming Li $^{4} \cdot$ Yi Jiang ${ }^{5} \cdot$ Peiya Wu ${ }^{1} \cdot$ Youzhu Li ${ }^{6} \cdot$ Weilin Peng ${ }^{1} \cdot$ Chengzhou Hua $^{1}$. \\ Yanfang Huang ${ }^{7} \cdot$ Huifang You ${ }^{8} \cdot$ Yuanyuan $\mathrm{Chen}^{9} \cdot{\text { Dianliang } \text { Lin }^{10} \cdot \text { Xiaoyu Yang }}^{8,11}$
}

Published online: 25 May 2021

(C) Society for Reproductive Investigation 2021

\section{Correction to: Reproductive Sciences https://orcid.org/10.1007/s43032-020-00429-7}

This article was update to correct the affiliation of Xiaoyu Yang.

Publisher's Note Springer Nature remains neutral with regard to jurisdictional claims in published maps and institutional affiliations.

Jianfeng Yao and Rongfu Huang contributed equally to this work.

The online version of the original article can be found at https://doi.org/ $10.1007 / \mathrm{s} 43032-020-00429-7$

Dianliang Lin

mqldl@163.com

$\triangle$ Xiaoyu Yang

yangxiaoyu683@163.com

1 Quanzhou Maternity \& Child Healthcare Hospital, Quanzhou, People's Republic of China

2 The School of Basic Medical Sciences, Fujian Medical University, Fuzhou, People's Republic of China

3 The Second Affiliated Hospital, Fujian Medical University, Quanzhou, People's Republic of China

4 Department of Histology and Embryology, Hunan University of Medicine, Huaihua, People's Republic of China

5 Quanzhou Center for Disease Control and Prevention, Quanzhou, People's Republic of China
6 The First Affiliated Hospital of Xiamen University, Xiamen, People's Republic of China

7 The First Affiliated Hospital, Fujian Medical University, Fuzhou, People's Republic of China

8 Fuzhou Hospital of Traditional Chinese Medicine Affiliated to Fujian University of Traditional Chinese Medicine, Fuzhou, People's Republic of China

9 Shuguang Hospital Affiliated to Shanghai University of Traditional Chinese Medicine, Shanghai, People's Republic of China

10 Fuzhou Maternity \& Child Healthcare Hospital, Fuzhou, People's Republic of China

11 Mengchao Hepatobiliary Hospital of Fujian Medical University, Fuzhou, People's Republic of China 